We report clinical course and genotype of four patients with hepatocerebral form of MDS.

Patients 1 and 2, daughters of consanguineous Roma parents, presented with liver failure at six and two days of life, respectively. The older sibling had lactic acidosis and progressive liver failure, without clear neurological involvement. Liver histology revealed gigantocellular hepatitis, fibrosis, cholestasis, hemosiderosis and steatosis. Working diagnosis was neonatal hemosiderosis. Despite the treatment, disease progressed to death at 40 days. Younger sibling had similar clinical course and MDS was suspected. Immunohistochemical staining of the deceased sibling's liver showed combined respiratory chain deficiency. Homozygous variant in the DGUOK gene in both patients confirmed the diagnosis. Despite cofactor/antioxidant treatment, patient 2 died at the age of two months.

Patient 3 presented with recurrent nonketotic hypoglycemia, cholestasis and hypotonia at the age of two months. Liver disease was slowly progressive, with permanently elevated lactate and alanine. Histology showed gigantocellular hepatitis, fibrosis, cholestasis, hemosiderosis, polymorphous mitochondria and microvesicular steatosis. MDS was suspected, but immunohistochemical staining was uninformative. Due to end-stage liver disease, LTx was performed at the age of six months. Patient died in early postoperative period. Whole exome sequencing (WES) revealed biallelic mutations in the MPV17 gene.

Patient 4 had intrauterine growth retardation, severe hypotonia and developmental delay since birth. Acute liver failure, presenting with ketotic hypoglycemia, lactic acidosis, hepatomegaly and coagulopathy, occurred at the age of four months. The individual additionally developed nystagmus. Brain MRI was normal. Liver biopsy showed steatosis and abnormal mitochondria. Immunohistochemistry and clearly decreased mtDNA copy number per nuclear genome in liver pointed to MDS. The disease progressed rapidly and patient died three weeks after admission. WES revealed two biallelic mutations in the POLG gene.

Revealing genetic basis of liver failure due to MDS, with WGS as an important option, is pre-requisite to decision on LTx. It is also essential for genetic counseling and prenatal diagnosis in future pregnancies.

\section{METHYLATION BIOMARKERS S-ADENOSYLMETHIONINE AND S-ADENOSYLHOMOCYSTEINE AND METHYLATION POTENTIAL IN NEWBORNS WITH CONGENITAL HEART DISEASE AND THEIR MOTHERS}

Dorotea Ninković*, Ksenija Fumić, Iva Bilandžija Kuš, Tarik Kapić, Ivana Križić, Danijela Petković Ramadža, Boris Filipović-Grčić, Vesna Benjak, Andrea Dasović Buljević, Ruža Grizelj, Ivo Barić. Department of Pediatrics, University Hospital Centre Zagreb, Zagreb, Croatia; School of Medicine, University of Zagreb, Zagreb, Croatia

10.1136/archdischild-2021-europaediatrics. 108

The low availability of folic acid or vitamin B12 in the early pregnancy and consequent mother's hyperhomocysteinemia are associated with the risk of having a child with congenital heart disease. The goal of this study was to determine whether homocysteine itself is a risk factor for congenital heart disease or changes in methylation biomarkers S-adenosylmethionine (AdoMet) and S-adenosylhomocysteine (AdoHcy) and methylation potential are responsible for a higher incidence of congenital heart disease.
In order to reach goals of this study, we included 127 newborns with congenital heart disease and 103 mothers of affected newborns with congenital heart disease. We measured AdoMet and AdoHcy in their plasma by high performance liquid chromatography tandem mass spectrometry and then calculate their methylation potential and compare them with the reference values.

In the group of newborns with congenital heart disease we have found statistically significant increased AdoMet compared to the referral group (289 vs. $184 \mathrm{nmol} / \mathrm{L}$ ). There was no statistically significant difference in the concentration of AdoHcy (67 vs. $81,5 \mathrm{nmol} / \mathrm{L}$ ). Methylation potential in this group was consequently statistically significantly higher (4,34 vs. 2,35). In the group of mothers of newborn with congenital heart disease we have found statistically significant increase of AdoHcy compared to the referral group (20 vs. $15,2 \mathrm{nmol} / \mathrm{L}$ ), but no statistically significant difference in the concentration of AdoMet (81 vs. 81,9 nmol/L). Methylation potential was statistically significantly lower (4,55 vs. 5,54$)$.

Increased concentrations of AdoMet or AdoHcy measured in our groups of examinees and statistically significant differences in the methylation potential values in our groups of examinees compared to the reference values point to changed methylation processes which could contribute to the pathogenesis of congenital heart disease. Further studies are needed to elucidate the exact mechanism by which disturbed methylation leads to higher risk for congenital heart disease and changes in methylation processes in the fetus.

\section{Neonatology}

\section{HEMIMEGALENCEPHALY AND OHTAHARA SYNDROME CAUSING NEONATAL SEIZURE - A CASE REPORT}

Katarina Pavičić Klancir*, Vlasta Đuranović, Sanja Pejić Roško, Milan Stanojević, Anita Pavičić Bošnjak, Ana Šimecki Butajla, Ivan Zovko. University hospital "Sveti Duh"

10.1136/archdischild-2021-europaediatrics. 109

Hemimegalencephaly (HME) is rare congenital, hamartomatuous malformation of the brain cortical development characterised by enlargement of all or a part of one cerebral hemisphere. It is estimated that prevalence range is from 1 to 3 cases per 1000 children with epilepsy, and 1-14\% among children with disturbed cortical development. Ohtahara syndrome is a rare infant epilepsy syndrome, characterised by "burst supression" pattern in EEG (high amplitude spikes followed by little brain activity or flattening of the brain waves). It is accompanied by severe neurodevelopemental delay, presumably caused by HME as a structural malformation.

We present a case of female premature newborn from bichorionic, biamniotic twin pregnancy, who was delivered vaginally after 35 weeks and 6 days, as the first twin. Apgar scores after 1 and 5 minutes were 10, physical examination was uneventful. The first in vitro fertilization resulted in twin pregnancy of 27-year healthy mother. Apart from oligohydramnios before delivery, pregnancy was uneventful. Family history of both parents was unremarkable. At the age of 24hours baby girl developed first tonic spasms lasting one minute and spontaneously ceasing, without losing consciousness. 
She received intravenous Phenobarbital $10 \mathrm{mg} / \mathrm{kg}$ followed by $5 \mathrm{mg} / \mathrm{kg} /$ day.

Initial laboratory findings including lumbal pucture and initial metabolic evaluation were all unremarkable. A tumor or congenital malformation of the left hemisphere of the brain was suspected after the first two dimensional brain ultrasonography. Multi Slice Computed Tomography (MSCT) revealed left HME, confirmed with the magnetic resonance imaging (MRI) together with polymicrogyria of frontal lobe, atypical form of the left Sylvian fissure and the left frontal ventriculomegaly. She developed refractory seizures (tonic; focal with automatisms - squelching, eye blinking; generalised, often waking her up from sleep). EEG showed suppression burst pattern and after extensive diagnostic evaluation the Ohtahara syndrome was diagnosed.

Despite several different antiepileptic drugs, and their different combination, frequency and severity of the seizures did not improve and she developed severe developmental delay. At the age of 10 months she underwent functional hemispherotomy, and so far, eight months after the surgery she experienced no seizures together with major improvement in neuromotor development (despite strabismus and right hemiparesis which occurred after surgery). Her twin sister is healthy, normally developing, without seizures. Our findings are in comply with the data from the literature, claiming that after surgery the improvement of the patients is remarkable.

\section{\begin{tabular}{|l|l}
\hline 110 & OPENING OF THE FIRST HUMAN MILK BANK IN \\
\hline
\end{tabular} CROATIA}

Branka Golubić Cépulićx, Anita Pavičić Bošnjak, Ivana Leskovar, Jurjana Novoselac, Koraljka Gojčeta, Vladimira Rimac, Ines Bojanić, Sanja Mazić. University Hospital Centre Zagreb, Department for Transfusion Medicine and Transplantation Biology, Croatian Tissue and Cell Bank, Human Milk Bank

\subsection{6/archdischild-2021-europaediatrics. 110}

Objective Foundation project for the first human milk banks (HMB) in Croatia was launched in 2017 as the result of a collaboration between the Ministry of Health, University Hospital Centre (UHC) Zagreb, UNICEF and the Croatian Association of Breastfeeding Support Groups. The HMB was opened in UHC Zagreb on November 15, 2019as a part of the Croatian Tissue and Cell Bank, a division of the Department of Transfusion Medicine and Transplantation Biology. The specific technical side is based on the PATH standards and Guide to the quality and safety of tissues and cells for human application, Council of Europe.

On January 10, 2020, HMB passed a Competent Authority (CA) inspection, conducted in accordance with the requirements of national tissue legislation that has been adapted to EU Directive 2004/23/EC on setting standards of quality and safety for the donation, procurement, testing, processing, preservation, storage and distribution of human tissues and cells. The specific technical part is based on the PATH standards and Guide to the quality and safety of tissues and cells for human application, Council of Europe.

The aim of this study was to review the very first experience in human milk banking in Croatia.

Methods We retrospectively analysed the data of the first 28 human milk donors.
Results From the opening to mid-February, 135 women showed interest in donating human milk, of which 28 became donors.

In February 2020, we had 24 still active donors. Four women stopped donating, with a median donation period of 2 months. All donors were tested for blood borne viruses by serology and NAT and were negative. In total, we received 79 $\mathrm{L}$ of donated human milk.We started pasteurizing the milk after obtaining a license. Of the 30 controlled pre-pasteurization milk pools, $10 \%$ was over the allowed microbial contamination. Microbiological controls were performed for each pasteurization cycle. They were all sterile. The nutritional values of milk were all within the expected range.

Conclusions Human milk is recognised as the optimal feeding for all newborn infants.

When mothers' own milk is not available, donor human milk provided by $\mathrm{HMB}$ is the second-best choice, especially for premature or sick infants. The opening of the HMB in Croatia is highly important in helping to provide the best possible medical care for prematurely born babies and infants with a serious medical condition when they cannot receive their mother's milk.

\section{MARKERS OF INFLAMMATORY RESPONSE OF THE INTESTINE IN NEWBORNS WHOSE MOTHERS RECEIVED A PROBIOTIC DURING THE 6 WEEKS BEFORE DELIVERY}

Margarita Gurova*, Podsvirova Elena, Romanova Tatyana., Popova ValentinaS. St. Petersburg State Pediatric Medical University

\subsection{6/archdischild-2021-europaediatrics.111}

To determine the concentration of the fecal eosinophil-derived neurotoxin

(EDN) and fecal calprotectin as intestinal markers of inflammatory response in newborns whose mothers received a probiotic during the 6 weeks before delivery Pregnant women $(n=115)$, depending on the number of detected chronic diseases, were divided into two groups. The main group included women with a history of two or more chronic diseases, including the genitourinary sphere

- 63/54.8\%. The comparison group included pregnant women without a history of chronic diseases or having one chronic disease, with the exception of the pathology of the urogenital sphere - 52/46.2\%. Pregnant women of the main group with a preventive purpose for 6 weeks before delivery used a probiotic containing Bifidobacterium longum $\square$ Streptococcus thermophiles. The concentration of fecal EDN and fecal calprotectin was assayed using an ELISA method (Immundiagnostik, Bensheim, Germany and Calprest, manufactured by Nycomed).

We found the following changes from the intestinal markers of inflammation in newborns depending on the use of probiotic by women before delivery: in children of the main group (mothers used probiotics) both markers were significantly lower than in comparison group (mothers not used probiotics).

Concentration of fecal EDN was $163.4 \pm 58.2$ vs 224.1 $\pm 83.4 \mathrm{ng} / \mathrm{g}(\mathrm{P}<0.001)$ and fecal calprotectin was 240.3 \pm 78.6 vs $315.6 \pm 101.2 \mathrm{mcg} / \mathrm{g}(\mathrm{P}<0.001)$. 